\title{
Standard Deviation of Spatially-averaged Surface Cross Section Data from the TRMM Precipitation Radar
}

\author{
Robert Meneghini, Senior Member, IEEE and Jeffrey A. Jones
}

\begin{abstract}
We investigate the spatial variability of the normalized radar cross section of the surface (NRCS or $\sigma^{0}$ ) derived from measurements of the TRMM Precipitation Radar (PR) for the period from 1998 to 2009 . The purpose of the study is to understand the way in which the sample standard deviation of the $\sigma^{0}$ data changes as a function of spatial resolution, incidence angle, and surface type (land/ocean). The results have implications regarding the accuracy by which the path integrated attenuation from precipitation can be inferred by the use of surface scattering properties.
\end{abstract}

Index Terms-surface cross section, NRCS, TRMM Precipitation Radar, atmospheric attenuation

\section{INTRODUCTION}

The Precipitation Radar (PR) aboard the TRMM (Tropical Rainfall Measuring Mission) satellite has provided nearly continuous, well-calibrated measurements of precipitation and surface scattering properties since the spacecraft launch in November, 1997. The radar operates at $13.8 \mathrm{GHz}$ with a cross-track scan through nadir out to $18^{\circ}$ consisting of 49 angles that comprise a swath of approximately $245 \mathrm{~km}$ from its present altitude of $400 \mathrm{~km}$. The orbital inclination of the satellite of $35^{\circ}$ is designed to provide precipitation information in the tropics and sub-tropics. A detailed description of the PR instrument and its operation is given by Kozu et al. [1].

For airborne and spaceborne precipitation radars, the surface reference technique (SRT) has shown itself to be useful for determining the path-integrated attenuation (PIA) through the atmosphere [2, 3]. The method rests on the assumption that $\sigma^{\mathrm{o}}$ is relatively constant under rain and rainfree conditions so that the apparent decrease in $\sigma^{\circ}$ in rainy conditions can be attributed to the path attenuation associated with precipitation along the radar beam. Over ocean, where wind speed and direction are the main determinants of $\sigma^{\circ}$, the reference data are generally taken over rain-free areas adjacent to the raining area at the same incidence angle. Over land,

Manuscript received April 8, 2010.

R. Meneghini is with NASA, Goddard Space Flight Center (phone: 301614-5652; fax: 301-614-5492; e-mail: Robert.Meneghini-1@ nasa.gov).

J. A. Jones is with Wyle Information Systems, Inc. (phone: 301-614-5721; fax: 301-614-5492; e-mail: Jeffrey.A.Jones-3@nasa.gov). where the surface is more heterogeneous, both spatial and temporal references have been used where by 'temporal' is meant the prior rain-free NRCS data over a fixed area and time period, classified with respect to incidence angle. Generalizations of the method have been proposed by the use of a $\sigma^{\circ}$ - wind speed model over ocean $[4,5]$ or by the use of cross-track fitting [6, 7]. In the standard TRMM Precipitation Radar (PR) algorithm, up to and including version 6 , a $1^{\circ}$ latitude-longitude grid has been used to store the monthly temporal statistics consisting of the sample mean, mean square, and number of samples of rain-free NRCS data for each of 25 incidence angles from nadir to $18^{\circ}$, combining data from the right- and left-hand sides of the scan. In the latest version, version 7 , planned to become operational in 2010 , the temporal reference data set has been replaced by $0.1^{\circ}$ grids for land, ocean and coastal backgrounds for each month using 12 years of TRMM PR data.

Although a $1^{\circ}$ resolution allows for a sufficient number of samples for a monthly estimate of mean and standard deviation at the various incidence angles, a concern is that this large averaging area includes heterogeneous targets. This heterogeneity would be responsible for some of the standard deviation of the sample thereby rendering the surface reference estimate unreliable for many rain events. Over ocean, very calm seas serve to enhance the near-nadir returns and reduce them for the larger off-nadir angles. If these calm-sea events are relatively rare, then an increase in the spatial resolution, while not reducing the average variance, would serve to separate the data into low and high variance regions. Since only those cells with a relatively low variance are applicable to the SRT, a greater fraction of the cells would qualify for the method.

The object of this study is to use 12 years of rain-free NRCS data from the TRMM Precipitation Radar (PR) to determine how the distribution of the sample standard deviation is affected by changing the grid size over which the statistics are gathered. We focus, in particular, on the behavior of the sample standard deviation (SSD) as a function of background type (land or ocean), incidence angle and the spatial resolution into which the data are averaged.

\section{SIMPLE EXAMPLE}

If the $\sigma^{0}$ data are such that the variation in $\sigma^{0}$ within a sub domain is much less than the change in the mean from one sub domain to the next, then we expect improvement in the sample 
variance of the gridded data as the cell size is decreased. This situation may hold over land areas with distinct boundaries separating, for example, crop-lands, forests, and bare soil.

To illustrate the type of improvement possible with higher spatial resolution, consider the following simple example of a square area divided along the diagonal into surface types $S_{1}$ and $S_{2}$ as shown in Fig. 1. We assume that the $\sigma^{0}$ data in $S_{1}$ are all the same and equal to $m_{1}$ and similarly for $S_{2}$ where the $\sigma^{0}$ are equal to $m_{2}$. Let the region be subdivided by a grid of size $\mathrm{N} \times \mathrm{N}$ so that the number of grid points that contain both surface types are the $\mathrm{N}$ cells along the diagonal. By assumption, the variance of $\sigma^{0}$ in the $\mathrm{N}(\mathrm{N}-1)$ cells outside the diagonal is zero. The sample variance of $\sigma^{0}$ in each of the $\mathrm{N}$ cells along the diagonal, assuming that a large and equal number of samples are taken from $S_{1}$ and $S_{2}$, is:

$$
\operatorname{var}\left(\sigma^{0}\right)=\frac{1}{4}\left(m_{1}-m_{2}\right)^{2}
$$

For large $\mathrm{N}$, the average standard deviation over the entire area $\left(\mathrm{N}^{2}\right.$ cells) is then

$$
\left\langle\operatorname{std}\left(\sigma^{0}\right)\right\rangle=\frac{\left|m_{1}-m_{2}\right|}{2 \sqrt{N}}
$$

i.e., the average sample standard deviation decreases as the square root of N. From the standpoint of the SRT, more important than the decrease in the average standard deviation is the fact that the fraction of cells that have a small variance (in this case zero) is $1-1 / \mathrm{N}$ so that the fraction of cases with useful reference data tends to 1 as the resolution increases.

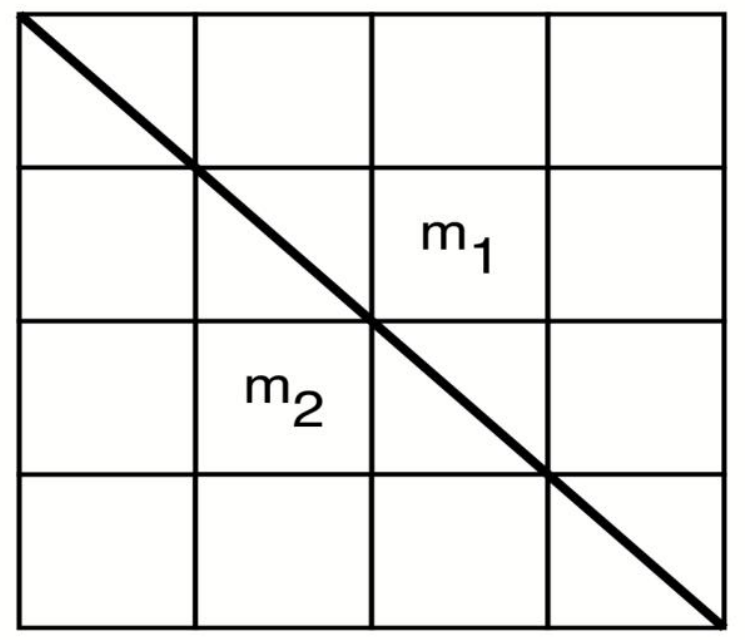

Fig. 1. Schematic of a region divided along the diagonal into two regions of constant NRCS. Only cells along the diagonal have non-zero variance.

The above example is relevant to the spatial variability in $\sigma^{0}$. However, in assessing the variability of the reference data, time as well as space must be considered. For example, over ocean, for very low wind speeds, the nadir return will be enhanced while the returns for off-nadir angles will be reduced. If these conditions occur infrequently, they will affect only a relatively small fraction of the high-resolution cells but will have an impact on the standard deviation of a larger fraction of the low-resolution cells. Thus, even though the average sample variance will be independent of resolution, as the resolution increases, the histogram of the sample variance will be broader so that a larger fraction of pixels will be associated with smaller variance. Whether this represents an improvement for application of the SRT depends on the relative magnitudes of the path attenuation and the standard deviation of $\sigma^{0}$.

For the SRT to be useful, the standard deviation of the rainfree $\sigma^{0}$ reference estimate should be less, and ideally much less, than the PIA. To get an idea of the magnitude of the PIA for the TRMM Precipitation Radar (PR), we use a kR relationship (where $\mathrm{k}$ is the specific attenuation in $\mathrm{dB} / \mathrm{km}$ and $\mathrm{R}$ is the rain rate in $\mathrm{mm} / \mathrm{h}$ ) that is relevant to stratiform rain at 20C [8]: $k=0.0245 R^{1.178}$. Using this relationship for a rain rate, $\mathrm{R}$, of $1 \mathrm{~mm} / \mathrm{h}$ over a $5 \mathrm{~km}$ path gives a two-way path integrated attenuation, PIA, equal to $0.245 \mathrm{~dB}$; for $5 \mathrm{~mm} / \mathrm{h}$ and $10 \mathrm{~mm} / \mathrm{h}$ the PIA increases to $1.63 \mathrm{~dB}$ and $3.69 \mathrm{~dB}$ respectively. These results imply that a $1 \mathrm{~dB}$ standard deviation in the reference data would give an unacceptably large relative error at $1 \mathrm{~mm} / \mathrm{h}$ but useful estimates at rain rates of $10 \mathrm{~mm} / \mathrm{h}$ and above.

\section{BINNING THE DATA}

Approximately every $0.6 \mathrm{~s}$, the PR completes a cross-track scan through nadir consisting of 49 fields of view from $-18^{\circ}$ to $+18^{\circ}$ in increments of $0.75^{\circ}$. With an effective 2-way beamwidth of $0.71^{\circ}$, the instantaneous field of view at the surface at nadir is approximately circular with a $5 \mathrm{~km}$ diameter from a $400 \mathrm{~km}$ altitude [1]. If no distinction is made between incidence angles on the left and right-hand sides of the swath, then the off-nadir surface returns, at incidence angles $0.75^{\circ} \times \mathrm{j}$ $(\mathrm{j}=1,24)$, are sampled twice every $0.6 \mathrm{~s}$ while the nadir surface return is sampled once during this interval. This implies that in the 12 years from 1998 to 2009 inclusive, about $6.3 \times 10^{8}$ samples were collected at nadir and twice this number for each of the off-nadir angles. For a $0.1^{\circ} \times 0.1^{\circ}$ grid, the highest resolution cell considered in this study, ranging from $37^{\circ} \mathrm{S}$ to $37^{\circ} \mathrm{N}$, the total number of cells is $740 * 3600=2.664 \times 10^{6}$. If the data are further subdivided by month, then the average number of counts per cell per month is about 20 at nadir and 40 for each of the off-nadir incidence angles. For the coarsest resolution grid considered in the study, $1^{\circ} \times 1^{\circ}$, the average count numbers for nadir and off-nadir increase to about 2000 and 4000 , respectively. To account for the change in $\sigma^{\circ}$ with surface type, separate arrays have been defined to store the data for ocean, land and coast backgrounds. A $\sigma^{\circ}$ measurement is classified as 'coast' if the instantaneous field of view (IFOV) of the PR contains both land and ocean backgrounds. Since the classification is done at the IFOV level, the total number of counts for any surface category will be the same, irrespective of the grid resolution. (It should be noted that rain cases, which account for about $4 \%$ of the data, 
are excluded from the surface reference data so the count number of rain-free data will be smaller than the calculations above would suggest.)

Histograms of the rain-free counts at nadir and at two offnadir angles for the high-resolution cells are shown in Fig. 2 for the month of October. Throughout the paper, we use results from the month of October since results from the other months are similar. These skewed distributions result from the fact that the satellite spends a greater proportion of the orbit near the $35^{\circ} \mathrm{N} / 35^{\circ} \mathrm{S}$ limits. (The extended sampling region, to $37^{\circ} \mathrm{N} / \mathrm{S}$, accounts for the additional coverage provided by the radar swath.) The results in Fig. 2 also show that there are cells in which no counts have been registered. A fraction of these zero-count cells arise from the regions between $36-37^{\circ} \mathrm{N}$ and $36-37^{\circ} \mathrm{S}$, another fraction from a region of northwestern Australia where the PR transmitter is turned off and a third, much smaller fraction, outside the regions just noted where no counts are registered.

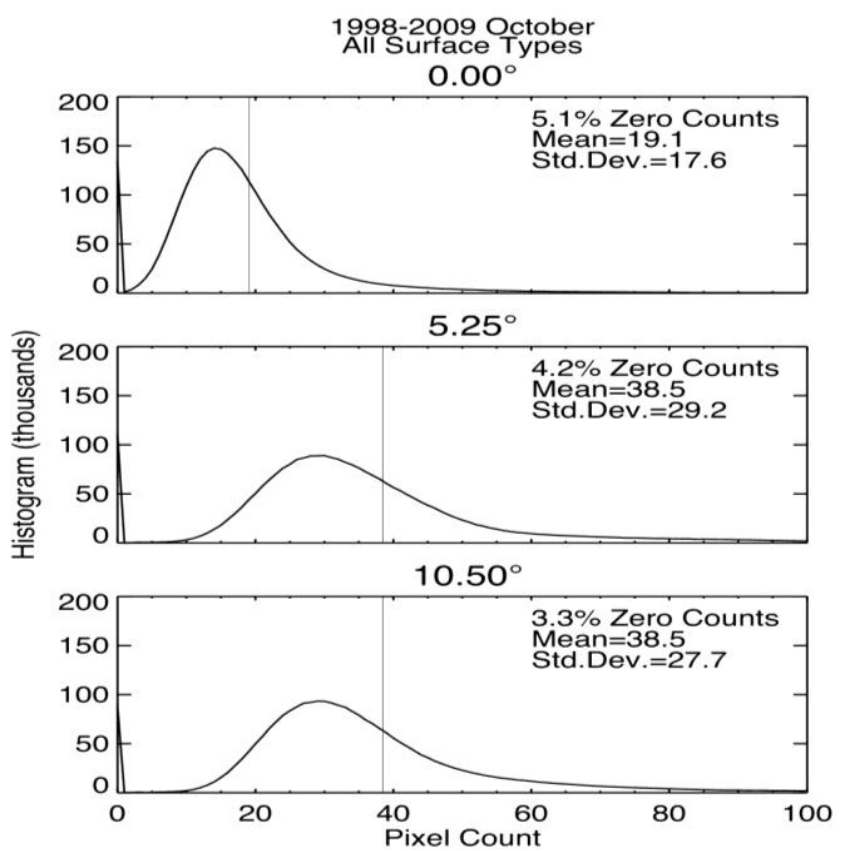

Fig. 2. Histograms of the number of non-raining counts (i.e., measurements of $\sigma^{0}$ ) over the $0.1^{\circ} \times 0.1^{\circ}$ grid for incidence angles of $0^{\circ}, 5.25^{\circ}$ and $10.5^{\circ}$ for 12 years of data (1998-2009) for the month of October.

\section{RESULTS}

Although the primary issue of interest is the change in the sample standard deviation with a change in spatial resolution, it is useful to consider the probability density functions (pdfs) of the sample means for different resolutions. Shown in Fig. 3 are the pdfs of the sample means for the high $\left(0.1^{\circ} \times 0.1^{\circ}\right)$ and low-resolution grids $\left(1^{\circ} \times 1^{\circ}\right)$ at 3 incidence angles over ocean for the month of October. As expected, in all three cases, the pdf of the sample mean at low resolution is more concentrated around the mean, with smaller variance. Although the pdf at $5.25^{\circ}$ incidence is fairly symmetric about the maximum for both resolutions, the pdf at nadir is skewed toward higher $\sigma^{\circ}$ values while the pdf at $10.5^{\circ}$ is skewed toward lower $\sigma^{\circ}$ values.
These long tails are associated with low wind speeds (calmer surfaces) that tend to increase the returns at near-nadir incidence and decrease them for higher incidence angles.

1998-2009 October

Ocean

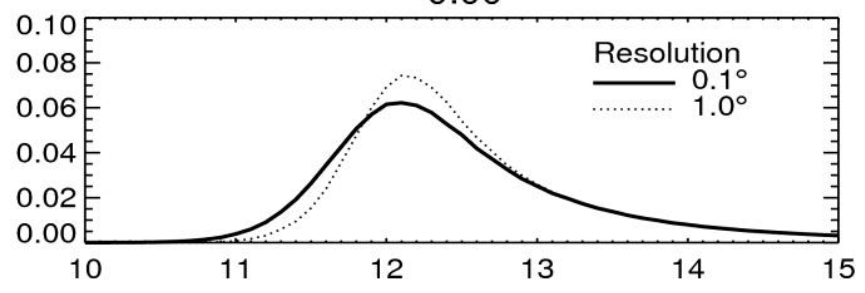

$5.25^{\circ}$

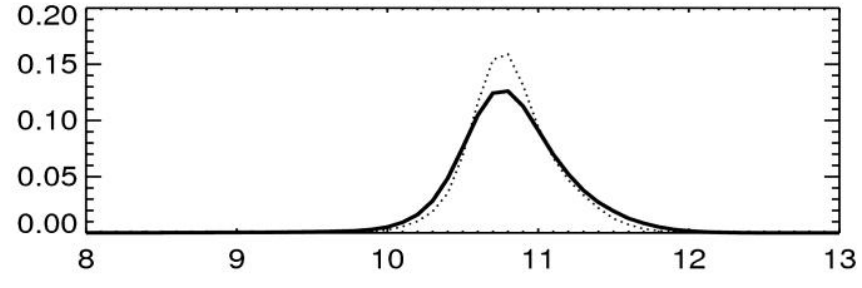

$10.50^{\circ}$

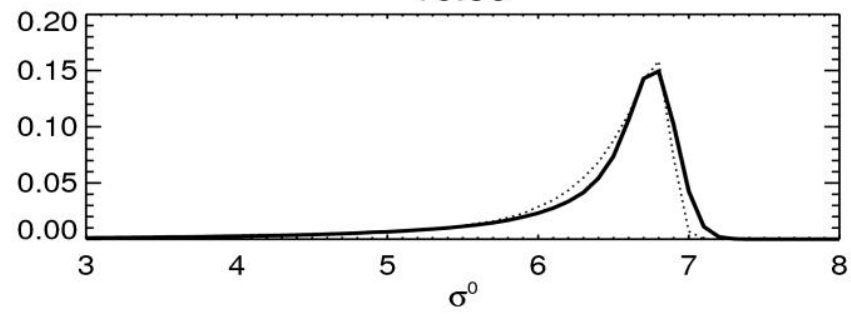

Fig. 3. Probability density functions of the sample means of $\sigma^{0}$ taken over $1^{\circ}$ and $0.1^{\circ}$ latitude-longitude cells over ocean at 3 incidence angles for the month of October.

Similar plots are shown in Fig. 4 for land background. Note that the variances of the pdfs are significantly broader for land than for ocean at the near-nadir incidence angles. The noisiness of the low resolution data arises from the relatively small number of land cells at the $1^{\circ} \times 1^{\circ}$ resolution and the fact that, at near-nadir angles, the sample mean varies over a much larger range of values than over ocean. As a consequence, there are fewer data points and greater sampling error at each bin.

Figs. 5 and 6 show the cumulative distribution functions (cdf) of the sample standard deviation of the gridded data for 4 different resolutions and 3 incidence angles for ocean (Fig. 5) and land (Fig. 6) backgrounds. Note that the cdf is defined as the probability that the random variable - in this case, the sample standard deviation (SSD) associated with the $\sigma^{0}$ data at each cell - is less than a given value. For example, from the top panel of Fig. 5, it can be seen that the probability that the SSD at the $0.1^{\circ} \times 0.1^{\circ}$ grid is less than $1 \mathrm{~dB}$ for about $45 \%$ of the cells and less than $2 \mathrm{~dB}$ for about $80 \%$ of the cells. In fact, for all SSD values of interest to the SRT (i.e., less about $2 \mathrm{~dB}$ ), it can be seen that at all incidence angles, and for ocean and land backgrounds, the fractional number of resolution cells below a given threshold increases as the resolution of the grid increases. 

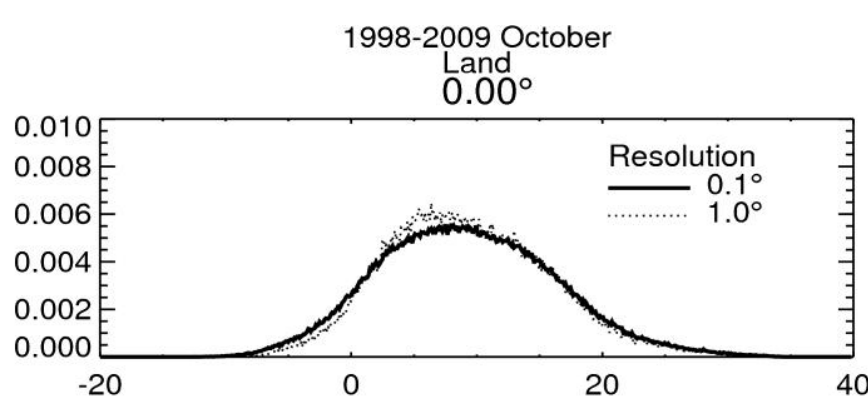

$5.25^{\circ}$
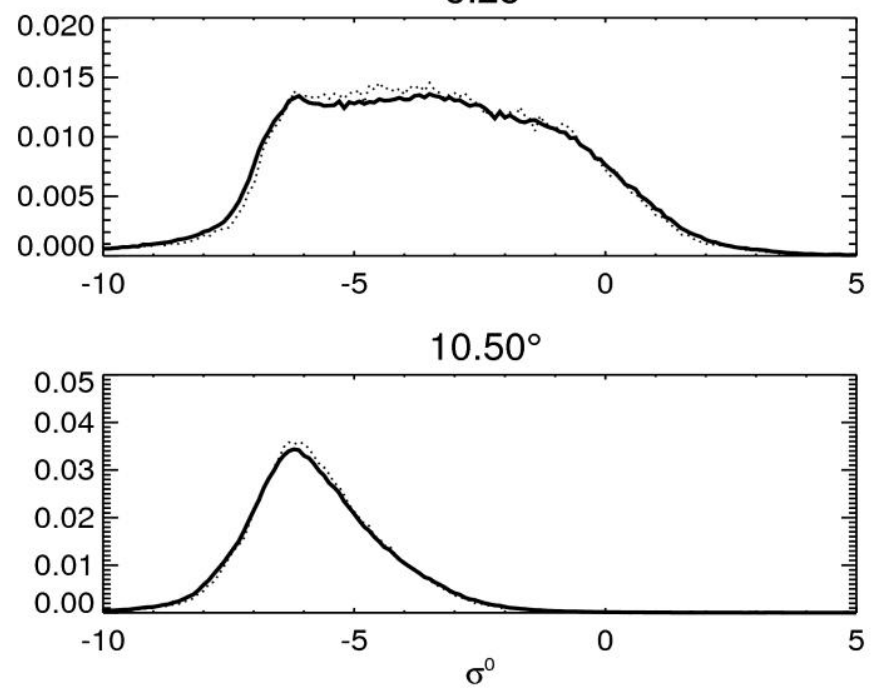

Fig. 4. Same as Fig. 3 but for land background

This improvement is shown in Fig. 7 for ocean and land backgrounds, where the fraction of cells with SSD less than 1 $\mathrm{dB}, \operatorname{Pr}(\mathrm{SSD}<1 \mathrm{~dB})$, is plotted versus incidence angle for spatial resolutions of $0.1^{\circ}, 0.25^{\circ}, 0.5^{\circ}$ and $1^{\circ}$. For both surface types and for all incidence angles, the results show that $\operatorname{Pr}(\mathrm{SSD}<1 \mathrm{~dB})$ increases as the resolution increases. For example, over ocean at $10.5^{\circ}$ the fractional area with SSD less than $1 \mathrm{~dB}$ increases from about $27 \%$ to $64 \%$ as the resolution is increased from a $1^{\circ}$ to a $0.1^{\circ}$ grid. Over land, at this angle, $\operatorname{Pr}(\mathrm{SSD}<1 \mathrm{~dB})$ increases from $20 \%$ to $44 \%$ with the same change in resolution. If we denote by $\rho$ the ratio $\operatorname{Pr}(\mathrm{SSD}<1$ $\left.\mathrm{dB}, 0.1^{\circ}\right) / \operatorname{Pr}\left(\mathrm{SSD}<1 \mathrm{~dB}, 1^{\circ}\right)$, i.e., the ratio of the fraction of cells with SSD less than $1 \mathrm{~dB}$ for the highest and lowest resolution cells, we find over ocean that $\rho>2$ for near-nadir angles (less than about $2^{\circ}$ ) and for off-nadir angles greater than about $9^{\circ}$. Over land, $\rho \geq 1.94$ for all angles where the ratio decreases nearly uniformly with angle with the smallest value, $\rho=1.94$, at the highest incidence angle $\left(18^{\circ}\right)$.

Plots of the mean sample standard deviation as a function of incidence angle and resolution are shown in Fig. 8 for ocean and land. As expected, the smallest value of SSD occurs at the highest resolution for all incidence angles for both land and ocean.
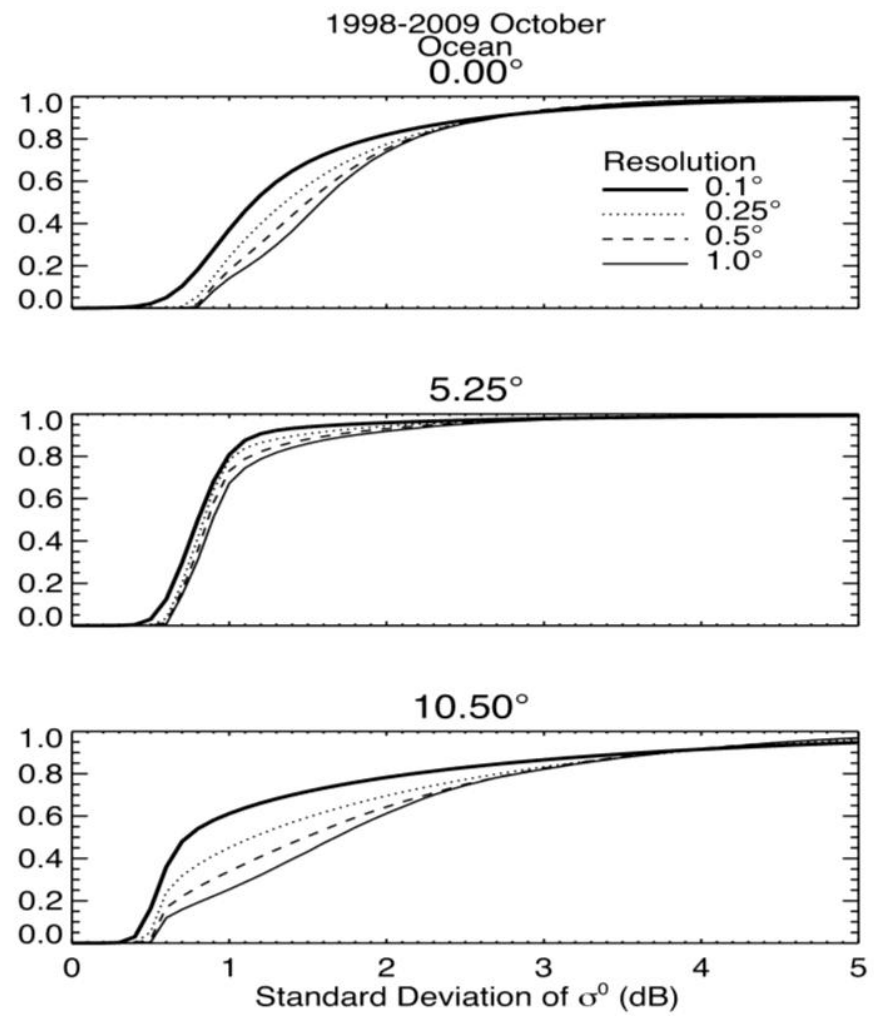

Fig. 5. Cumulative distributions of the sample standard deviation of $\sigma^{0}$ for ocean background at 4 spatial resolutions for 3 incidence angles for measurements in 1998-2009, inclusive, for the month of October.
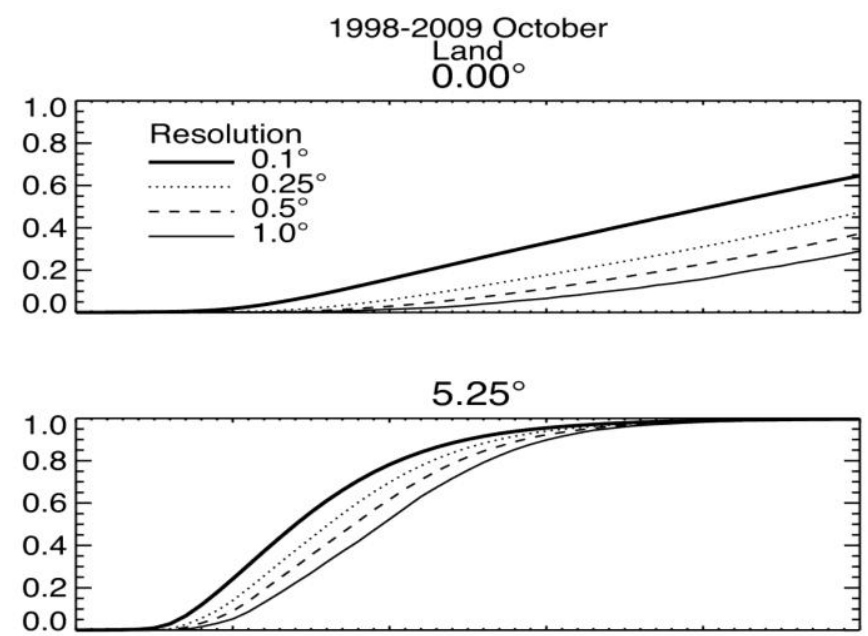

$10.50^{\circ}$

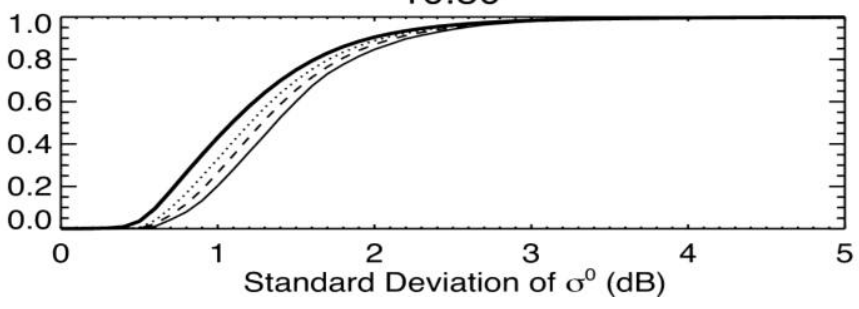

Fig. 6. Same as Fig. 5 but for land. 

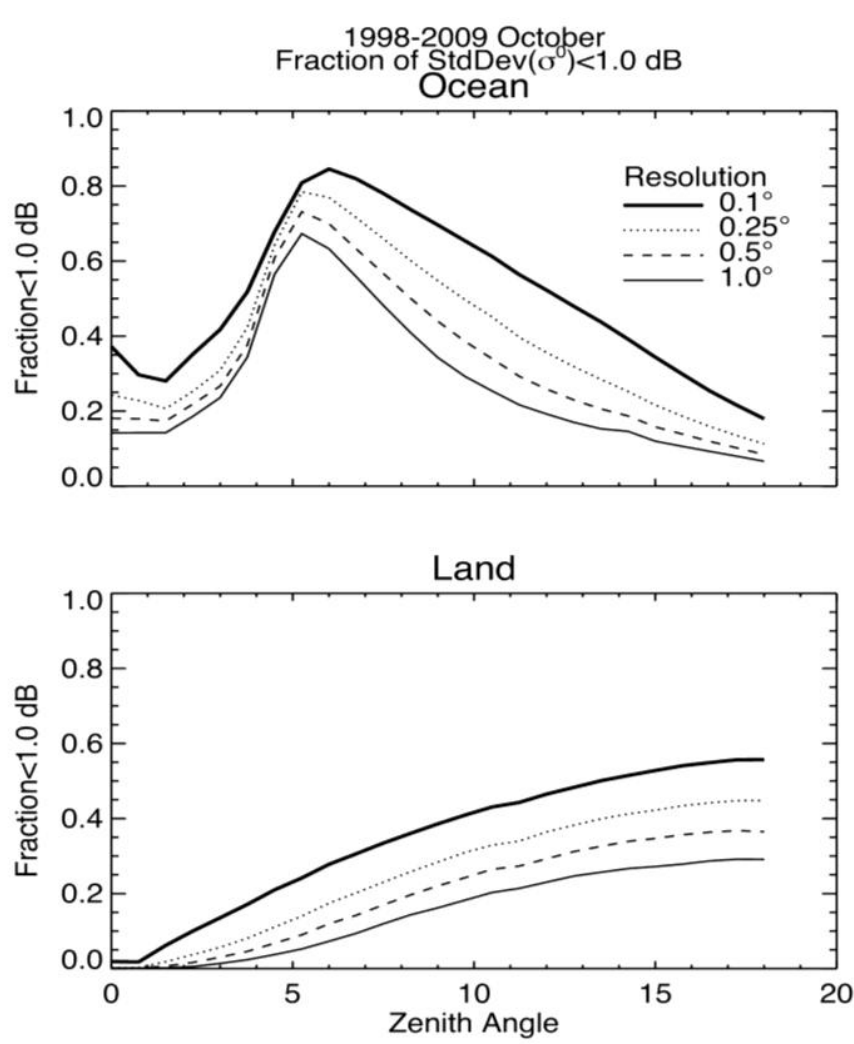

Fig. 7. Fraction of cells for which the sample standard deviations is less than $1 \mathrm{~dB}$ for ocean (top) and land (bottom) as a function of incidence angle for 4 resolutions.
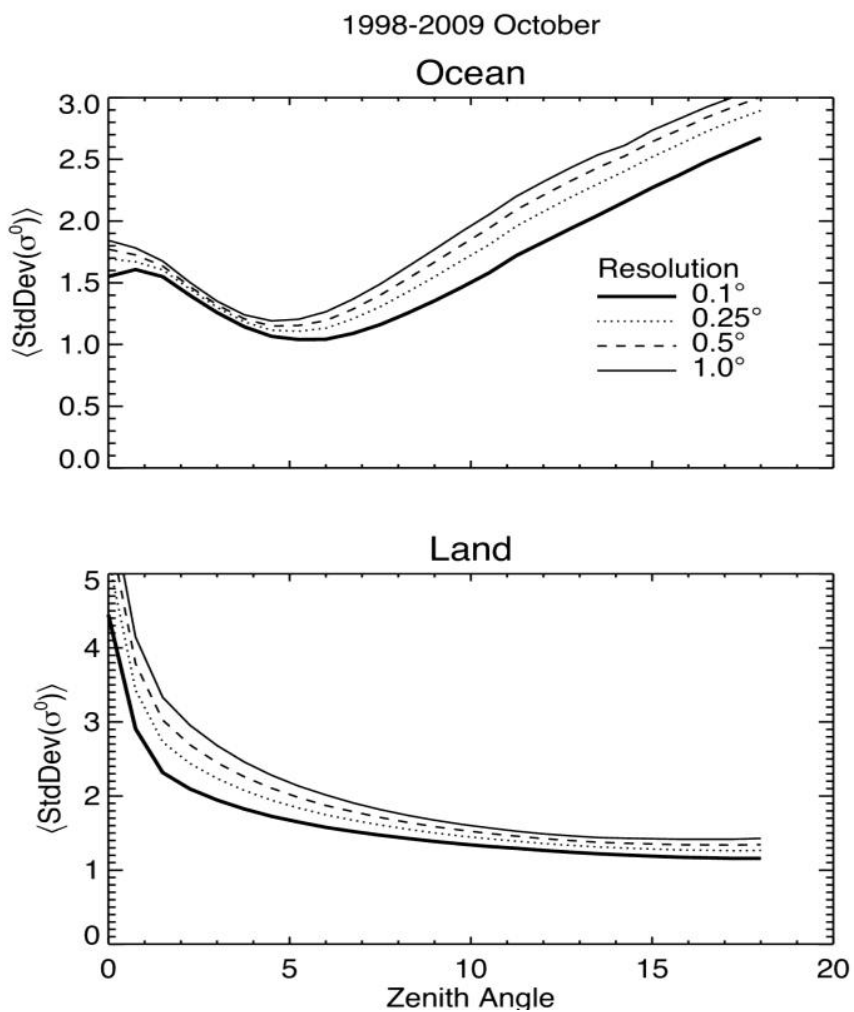

Fig. 8. Sample standard deviation of $\sigma^{0}$ versus incidence angle for 4 resolutions for ocean (top) and land (bottom).

\section{SUMMARY}

The accuracy of surface reference technique, a method to estimate the path attenuation associated with precipitation, depends on the variability of the normalized radar cross section, $\sigma^{0}$, of the surface under rain-free conditions. The objective of the investigation is to assess how the variability in the spatially averaged $\sigma^{0}$ data changes as a function of incidence angle, surface type and spatial resolution. The results show that the mean sample standard deviation (SSD) of the TRMM PR $\sigma^{\circ}$ data decreases at angles between $0^{\circ}$ and $18^{\circ}$, over land and ocean as the spatial resolution is changed from $1^{\circ}$ to $0.1^{\circ}$. More importantly, for the application of the surface reference technique, the fraction of resolution cells over land with SSD values below $1 \mathrm{~dB}$ increases by nearly a factor of 2 or more at all angles when the averaging area is changed from $1^{\circ}$ to $0.1^{\circ}$. Over ocean, the fractional number of cells with SSD less than $1 \mathrm{~dB}$ increases by more than a factor of 2 for near nadir (less than $2^{\circ}$ ) and off-nadir angles greater than about $9^{\circ}$ with a change in resolution from $1^{\circ}$ to $0.1^{\circ}$. Since the variance in the path-attenuation estimate is directly proportional to the variance in the $\sigma^{0}$ reference estimate, these results suggest that the accuracy of the attenuation estimate, when using this type of reference data, can be improved by using a higher resolution spatial grid.

\section{REFERENCES}

[1] T. Kozu et al., "Development of Precipitation Radar onboard the Tropical Rainfall Measuring Mission (TRMM) satellite," IEEE Trans. Geosci. Remote Sens., vol. 39, pp. 102-116, 2001.

[2] R. Meneghini, J. Eckerman, and D. Atlas, "Determination of rain rate from a spaceborne radar using measurements of total attenuation," IEEE Trans. Geosci. Remote Sens., vol. 21, pp. 34-43, 1983.

[3] R. Meneghini, T. Iguchi, T. Kozu, L. Liao, K. Okamoto, J.A. Jones, and J. Kwiatkowski, "Use of the surface reference technique for path attenuation estimates from the TRMM Precipitation Radar," J. Appl. Meteor., vol. 39, pp. 2053-2070, 2000.

[4] L. Li, E. Im, L.N. Connor, and P.S. Chang, "Retrieving ocean surface wind speed from the TRMM precipitation radar measurements," IEEE Trans. Geosci. Remote Sens., vol. 42, pp. 1271-1282, 2004.

[5] S. L. Durden, L. Li, E. Im and S.H. Yueh, “A surface reference technique for airborne Doppler radar measurements in hurricanes," J. Atmos. Oceanic Technol., vol, 20, 269-275, 2003.

[6] R. Meneghini, J.A. Jones, T. Iguchi, K. Okamoto, and J. Kwiatkowski, "A hybrid surface reference technique and its application to the TRMM Precipitation Radar," $J$. Atmos. Oceanic Technol., vol. 21, pp. 1645-1658, 2004.

[7] S. Seto and T. Iguchi, "Rainfall-induced changes in actual surface backscattering cross sections and effects on rain-rate estimates by spaceborne Precipitation Radar," $J$. Atmos. Oceanic Technol., vol 24, pp. 1693-1709, 2007.

[8] T. Iguchi, T. Kozu, J. Kwiatkowski, R. Meneghini, J. Awaka, and K. Okamoto, "Uncertainties in the rain profiling algorithm for the TRMM Precipitation Radar," $J$. Meteor. Soc. Japan, vol. 87A, pp. 1-30, 2009. 
'Standard Deviation of Spatially-averaged Surface Cross Section Data from the TRMM Precipitation Radar'

Robert Meneghini and Jeffrey A. Jones

One way of estimating path-integrated attenuation (PIA) through the precipitation is by using the surface as a reference target. The idea is to compare the radar surface return in the presence and absence of rain; the difference between these two measurements provides an estimate of the PIA. This concept has been implemented as one of the standard algorithms used in the TRMM Precipitation Radar (PR) processing. However, when we calculate the variability of the surface return under rain-free conditions, we see a significant amount of variability, particularly at nearnadir angles over land and off-nadir angles over ocean. We find that by reducing the region over which the rain-free surface data are averaged from a $1 \times 1$ degree (latitude-longitude) resolution to a 0.1 by 0.1 degree resolution that the standard deviation of the rain-free surface return data decreases. This result implies that we can obtain a more stable reference, and thereby more accurate estimates of path attenuation, by using a higher resolution averaging of the rain-free surface return data. 